\title{
Diversidad de Araceae en el paisaje forestal del noroccidente del Ecuador
}

\section{Diversity of Araceae in the northwestern forest landscape of Ecuador}

\author{
Marlon Núñez Castro ${ }^{1 *}$, Gladys Benavides Ordoñez ${ }^{2}$, Eduardo Argotti Valencia ${ }^{2}$, Marco Heredia Rengifo ${ }^{1,3}$, \\ Rolando López-Tobar ${ }^{4}$ \\ ${ }^{1}$ Facultad de Ciencias de la Vida, Universidad Estatal Amazónica (UEA), Pastaza 160101, Ecuador \\ ${ }^{2}$ Investigadores independientes, Ecuador \\ ${ }^{3}$ Programa de Economía de Recursos Naturales y Desarrollo Empresarial, UEA, Ecuador \\ ${ }^{4}$ Carrera de Ingeniería Forestal, Universidad Técnica Estatal de Quevedo, UTEQ, Ecuador \\ *Correspondencia: mnunez@uea.edu.ec
}

Rec.: 02.04.2019. Acept.: 31.10.2019.

Publicado el 30 de diciembre de 2019

\begin{abstract}
Resumen
A raceae en Ecuador tiene gran endemismo. Se estima que en las estribaciones occidentales de Los Andes, se podrían encontrar especies que desaparecerían antes de ser descritas. De acuerdo a la UICN, el alto porcentaje de este endemismo se encuentra bajo alguna categoría de amenaza. El objetivo es evaluar la diversidad y ecología de la familia Araceae, como potencial para complementar políticas de conservación de los bosques de esta región. La investigación se la realizó en: 1. Bosque de Sarapullo, 2. Bosque de la Comunidad Tsáchila Peripa y, 3. Bosque Protector La Perla. Se implementaron 10 transectos de $50 \times 2 \mathrm{~m}$ en cada sector. Los resultados muestran que la abundancia de especies fue similar entre Sarapullo y La Perla, pero en el Sector Peripa fue inferior a las dos, cabe anotar que este sector, ha sido sometido a extracción selectiva por parte de la comunidad Tsáchila, aunque aquí se presentó la mayor diversidad. Existe mayor similitud entre Peripa y La Perla que podría ser debido a que se encuentran cercanas geográfica y altitudinalmente, mientras que entre los pares Sarapullo-Peripa y Sarapullo-La Perla la similitud fue menor debido a la gran diferencia en estas dos variables. Algo similar ocurre con las especies compartidas entre Peripa y $\mathrm{La}$ Perla que es mayor comparando cualquiera de ellas con Sarapullo. Los géneros de hábitos hemiepífitos y epífitos se presentan en mayor porcentaje frente a los géneros de hábitos terrestres, probablemente los fustes brindan mayor garantía de supervivencia. Adicionalmente se encontraron 8 especies endémicas compartidas entre los tres sectores y 3 especies nuevas aún por confirmar. Las colecciones botánicas, reposan en Herbarios de Ecuador y de Estados Unidos.
\end{abstract}

Palabras clave: Endemismo, diversidad, abundancia, similitud, comunidad Tsáchila.

\begin{abstract}
A raceae in Ecuador has great endemism. It is estimated that in the western foothills of the Andes, species that would disappear before being described could be found. According to IUCN, the high percentage of this endemism is under some category of threat. The objective was to evaluate the diversity and ecology of the Araceae family, as a potential to complement forest conservation policies in this region. The investigation was carried out in: 1. Sarapullo Forest, 2. Tsáchila Peripa Community Forest and, 3. La Perla Protective Forest. 10 transects of $50 \times 2 \mathrm{~m}$ were implemented in each sector. The results show that the abundance of species was similar between Sarapullo and La Perla, but in the Peripa Sector it was less than two, it should be noted that this sector has undergone selective extraction by the Tsáchila community, although it was presented here the greatest diversity. There is greater similarity between Peripa and La Perla that could be due to the fact that they are geographically and altitudinally close, while between the Sarapullo-Peripa and Sarapullo-La Perla pairs the similarity was less, due to the large difference in these two variables. Something similar occurs with the species shared between Peripa and La Perla, which is larger by comparing any of them with Sarapullo. The genus of hemiepiphyte and epiphytic habits are presented in a greater percentage compared to the genres of terrestrial habits, the shafts probably provide a better guarantee of survival. Additionally, 8 endemic species were shared between the three sectors and 3 new species still to be confirmed. Botanical collections rest in Herbariums of Ecuador and the United States.
\end{abstract}

Keywords: Endemism, diversity, abundance, similarity, Tsáchila community. 


\section{Introducción}

$\mathrm{L}$ a Flora del Ecuador incluye 18.198 especies de plantas vasculares y 1663 de plantas no vasculares, aproximadamente 5400 son consideradas endémicas. $\mathrm{Su}$ estado de conservación sigue el mismo patrón alarmante observado en la edición anterior del Libro rojo. En el que se menciona que la mayoría de endémicas $(78 \%$ 3508 especies), están amenazadas en algún grado.

Para Araceae se sugiere que el $42.13 \%$, es decir 67 de sus 159 especies endémicas están amenazadas; 11 En Peligro Crítico, 26 En Peligro y 30 Vulnerables; una proporción muy alta (45\%) están en la categoría Datos Insuficientes, mientras 13 son Casi Amenazadas y apenas 7 en preocupación menor. (Léon-Yánez et al., 2011). Del total de Araceae, el género Anthurium cuenta con 291 especies, más de la mitad son endémicas. El sector de Sarapullo, presenta el ecosistema: Bosque siempre verde montano de la Cordillera Occidental de los Andes, catalogado como uno de los más ricos, y más presionados del Neotrópico, de acuerdo con (MAE, 2015), es uno de los 5 ecosistemas con mayor número de especies de flora, (3053), con alto grado de endemismo (420), pero a la vez, uno de los de mayor número de especies de flora en categoría de amenaza (235).

Estas evidencias de amenaza se presentan principalmente por la degradación de los bosques, la falta de información de la diversidad de los ecosistemas y su estado de conservación y los procesos de deforestación, que según (Sierra, 2013) son un elemento dinámico de un paisaje en el que se observan cambios constantes de un uso a otro; la deforestación bruta para el periodo 2008-2014 se encontraba en 97917 ha/año, valor muy alto en relación a la superficie del país.

Se considera que la flora completa de Araceae tendría aproximadamente 1500 especies, con cientos aún por describir especialmente en las en las estribaciones occidentales de la cordillera de Los Andes, entre los 400 y $1800 \mathrm{~m}$ de altitud. (Léon-Yánez et al., 2011). En estas zonas montañosas con elevaciones intermedias, Araceae manifiesta gran endemismo, incluso por encontrarse en parte dentro del Corredor de Conservación ChocóManabí, con presencia de centros de diversidad para la familia. (Ganzenmüller, 2010). Abonando a esta diversidad en la zona, Benavides (1992a) en el estudio taxonómico de Araceae en la reserva forestal "La Favorita" (bosque muy húmedo Montano bajo), encontró 7 géneros y 29 especies.

Araceae, presenta adaptaciones y modos de vida específicos que representan indicadores de determinadas condiciones ecológicas, así los tipos de hábitats ocupados por Araceae son mucho más grandes que en la mayoría de las familias (Madison, 1978b), encontrándose especies tanto epífitas como terrestres y hemiepífitas; sin embargo, el porcentaje más grande de Araceae, son epífitas. Algunos géneros como Anthurium y Stenospermation son terrestres, pero usualmente llegan a ser epífitas verdaderas, mientras que otros géneros como Monstera, Syngonium y la mayoría de las especies de Philodendron, comienzan su ciclo de vida en el suelo y luego trepan a los árboles, convirtiéndose en hemiepífitas y perdiendo en parte su conexión con el suelo, para convertirse en verdaderas epífitas.

Araceae también están considerada como un recurso no maderable importante en el manejo de los bosques, con utilización alimenticia, medicinal y ornamental como los Anturios que adornan jardines (Add van Uffelen, 1996). En la Amazonía (Cerón, 1993) y (Alarcón, 1984) mencionan utilidad medicinal para los géneros Anthurium, Philodendron, Syngonium, Urosphata, y alimenticio para Colocasia, Monstera y Xanthosoma. Sin embargo, el manejo de esta biodiversidad local tiene deficiencias, por lo que los bosques, pueden reducirse a fragmentos cada vez más explotados y aislados, como es el caso del Bosque de Peripa, limitando así, el importante potencial de Araceae.

\section{Materiales y métodos}

\section{Sitios de la investigación}

Se efectuó en Ecuador en tres sectores a diferentes gradientes altitudinales: Sector 1. Bosques de Sarapullo $00^{\circ} 08^{\prime} 36^{\prime \prime}$ S, 7843'74" W. Sector 2. Comunidad Tsáchila de Peripa $00^{\circ} 09^{\prime} 34^{\prime \prime}$ S, $078^{\circ} 28^{\prime} 43^{\prime \prime}$ W y Sector 3. Bosque Protector La Perla $00^{\circ} 00^{\prime} 09^{\prime}$ 'S, $079^{\circ} 22^{\prime} 53^{\prime}$ 'W.

Sector 1. El bosque de Sarapullo, declarado como bosque protector el 26 de junio de 1986, tiene una superficie de 21585 ha, ubicado entre las provincias de Pichincha y Cotopaxi; su altitud va de 1700 a 2100 msnm, su precipitación anual está entre 2000 a 3000 $\mathrm{mm}$, con temperaturas entre 18 a $22^{\circ} \mathrm{C}$. De acuerdo con (MAE, 2013), corresponde al bosque siempre verde Montano Bajo y bosque de neblina Montano.

Composición florística. Parte de este sector pertenece al Bosque Protector Toachi-Pilatón, que cuenta con alta representatividad de bosque primario, tanto de ceja andina como de bosque nublado, en los de declives de la cordillera Occidental. El estrato superior cuenta con árboles de 20 a 30 metros, gran variedad de epífitas y trepadoras de Bromeliaceae, Orchidiaceae, Araceae, Polypodiaceae. Según (Gómez \& Toaza, 1997), en 0.1 ha se encontraron 323 árboles con DAP $\geq 2.5 \mathrm{~cm}$, de 75 especies y 36 familias. Aunque se considera que esta diversidad es baja con relación a otros estudios en sitios cercanos como Chiriboga 149 sp. (Cerón, 1993), Reventador 130 sp. (Cerón, 1973). 


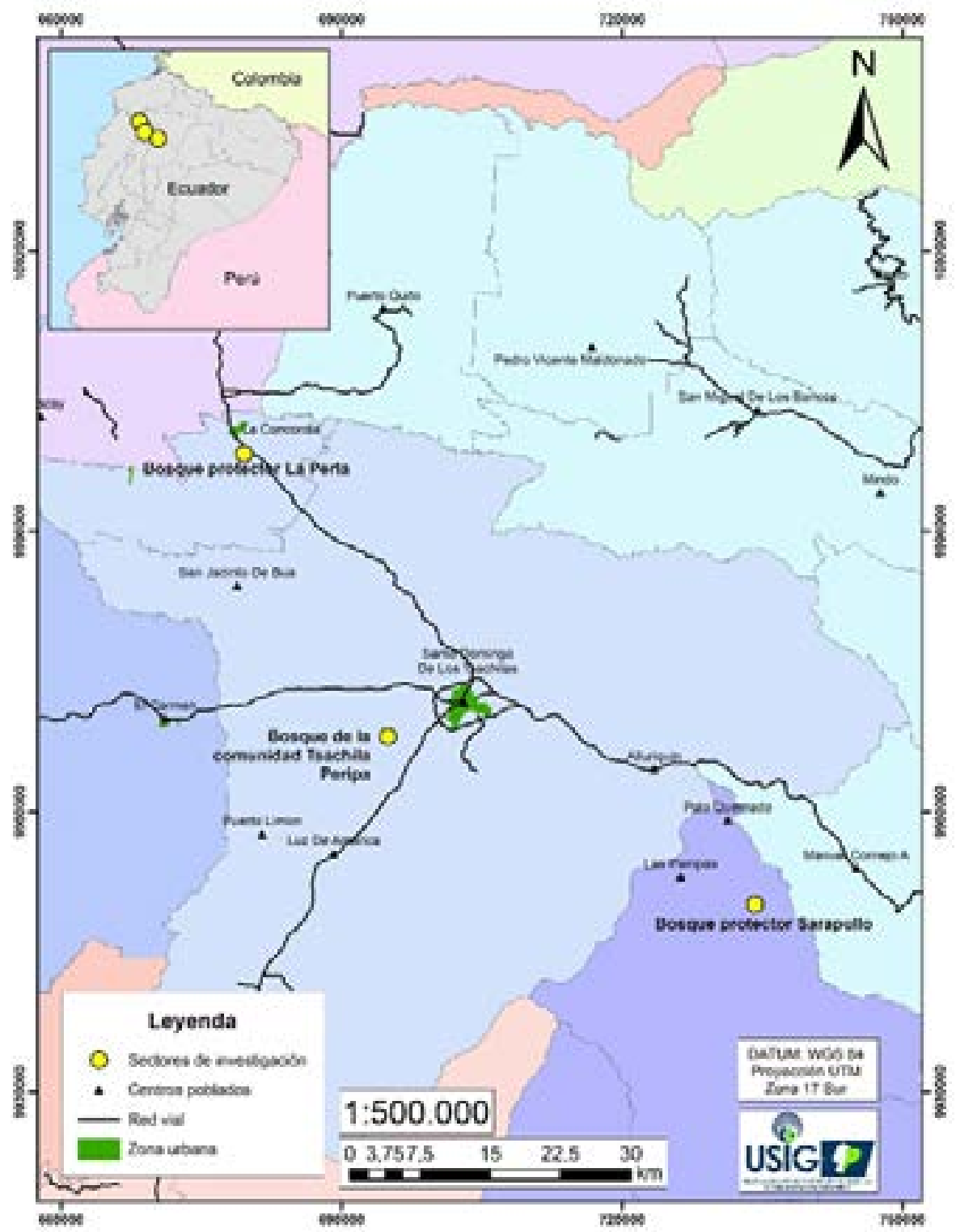

Figura 1. Sectores de la investigación (a) Bosque Protector La Peña, (b) Bosque de la comunidad Tsáchila Peripa, (c) Bosque Protector Sarapullo

Sector 2. El Bosque Protector La Perla, declarado así el 11 de agosto de 1986, posee 250 hectáreas de bosque nativo, ubicado a $42 \mathrm{~km}$ al noroeste de Santo Domingo de los Colorados, su altitud promedio es de $360 \mathrm{msnm}$, de acuerdo (MAE, 2013), corresponde al Bosque siempre verde de tierras bajas del chocó ecuatorial. La humedad relativa es alta cerca del $80 \%$. El área tiene una pendiente de $0^{\circ}$ a $12^{\circ}$, su clima es tropical húmedo, con temperatura media de 24 a $44^{\circ} \mathrm{C}$, humedad relativa promedio de $88 \%$ y precipitación anual promedio de $3 \mathrm{~m}^{3} / \mathrm{m}^{2}$ de suelo.

Composición florística. El sector está dividido en 18 sistemas ecológicos diferentes, 17 de ellos en el área de selva natural. Los más importantes están a orillas del rio Cucaracha, a esta zona se le ha denominado área intangible y representada el $40 \%$ de la superficie protegida. (EcoCiencia, 1986) (PDOT).

Sector 3. El Bosque de la comunidad Tsáchila Peripa se ubica en el km. 7 de la vía Santo Domingo - Quevedo, su altitud varía de 400 a $600 \mathrm{msnm}$ con temperaturas de $18 \mathrm{a} 24^{\circ} \mathrm{C}$ y precipitaciones anuales de 2000 a $3000 \mathrm{~mm}$, de acuerdo con (MAE, 2013), corresponde al bosque siempre verde Montano Bajo y bosque de neblina Montano. 
Composición florística. El bosque se encuentra alterado, presenta regeneración de especies de rápido crecimiento, aunque se pueden ver todavía algunos árboles originales, con gran variedad de herbáceas tanto epifitas como trepadoras y bejucos. El estrato superior alcanza una altura de 20 a $30 \mathrm{~m}$, aunque existen algunas especies emergentes que sobrepasan estas alturas, dentro de las familias Arecaceae, Boraginaceae, Bombacaceae, Burseraceae, Cecropiaceae, Verbenaceae y Ulmaceae. A pesar de la alteración se han registrado en 0.1 ha, 268 individuos correspondientes a 81 especies y 37 familias, sin embrago esta diversidad puede ser considerada de mediana a baja, en relación con estudios efectuados en zonas de altitud similar, tales como: Río Palenque, 119 sp. (Dodson \& Gentry, 1978); Jauneche, 96 sp. (Dodson et al. 1985), (Gómez \& Toaza, 1997).

\section{Metodología}

Se realizaron inicialmente colecciones al azar (prueba Sinicial de presencia de especies de Araceae) y luego se aplicaron transectos de $50 \times 2 \mathrm{~m}$ (10 por cada sector: $3000 \mathrm{~m}^{2}$ de muestreo en total). Para las colecciones se tomaron en cuenta las normas establecidas para Araceae (Croat 1985) y las muestras fueron procesadas hasta especímenes de herbario.

Análisis matemáticos y estadísticos. Se estableció el área mínima de muestreo, se realizaron mediciones de Abundancia total, por géneros, especies y sectores, se calcularon la Frecuencia de especies por sector, la Densidad, los Índices de diversidad (Simpson y
Shannon), las curvas de Abundancia-Diversidad, las Especies dominantes por sector, la Similitud entre sectores (coeficiente de Sorensen y el índice de Morisita) y finalmente la similitud entre transectos y sectores mediante un Dendrograma de frecuencias basado en los 30 transectos analizados.

\section{Resultados y discusión}

Área mínima: La saturación de especies para los tres sectores, se estableció al cubrir un área de $800 \mathrm{~m}^{2}$ (superficie acumulada de 8 transectos), esta es el área en la cual se han registrado para cada sector todas o la gran mayoría de especies de esta familia. (Figura 2)

Abundancia total de Araceae. La estructuración florística de Araceae mostró un gradiente diferencial entre los sectores: La Perla difirió sustancialmente de Sarapullo, Peripa y la Perla mostraron similitud, en tanto Sarapullo y Peripa mostraron diferencias.

Sarapullo. Se encontraron 34 especies en 7 géneros (Anthurium, Philodendron, Stenospermation, Chlorospatha, Rodospatha, Monstera y Xanthosoma). El género con mayor representatividad fue Anthurium (816); la especie más abundante de hábito hemiepífitoepífito fue Philodendron sulcatum K. Krause.

Peripa. Se encontraron 36 especies en 8 géneros (Anthurium, Philodendron, Chlorospatha, Rodospatha, Monstera, Xanthosoma, Dieffenbachia y Syngonium). El género con mayor representatividad fue Philodendron (392), las

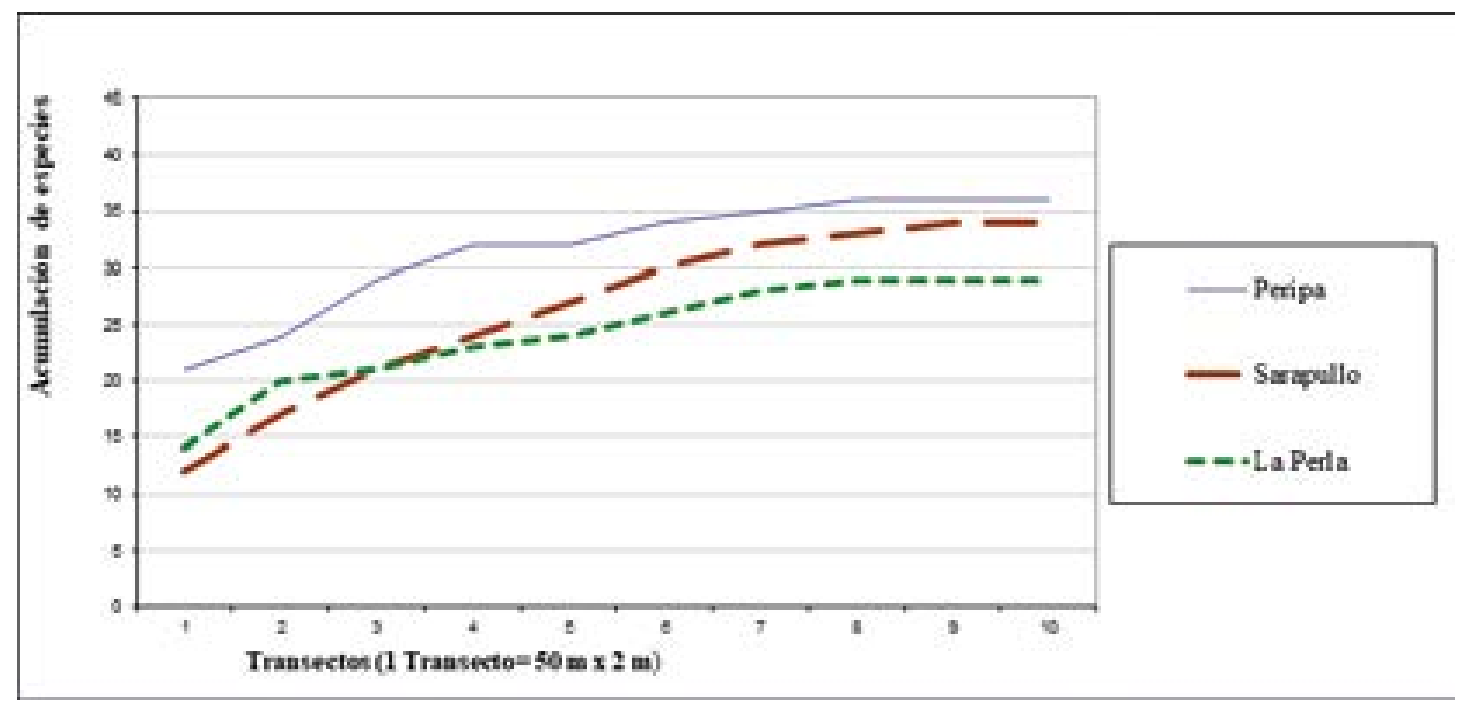

Figura 2. Acumulación de especies en los tres sectores (a) Peripa, (b) Sarapullo y (c) La Perla en un área de $800 \mathrm{~m}^{2}$ 
Cuadro 1. Abundancia total de los géneros de Araceae en los sectores Sarapullo, Peripa y La Perla

\begin{tabular}{|c|c|c|c|c|c|c|c|}
\hline \multirow{2}{*}{ Géneros } & \multicolumn{2}{|c|}{ Sarapullo } & \multicolumn{2}{|c|}{ Peripa } & \multicolumn{2}{|c|}{ La perla } & \multirow{2}{*}{$\begin{array}{c}\mathbf{N}^{\circ} \\
\text { Total de } \\
\text { Individuos }\end{array}$} \\
\hline & $\%$ & $\begin{array}{c}\mathrm{N}^{\circ} \\
\text { Individuos }\end{array}$ & $\%$ & $\begin{array}{c}\mathrm{N}^{\circ} \\
\text { Individuos }\end{array}$ & $\%$ & $\begin{array}{c}\mathrm{N}^{\circ} \\
\text { Individuos }\end{array}$ & \\
\hline Anthurium & 54.66 & 816 & 25.53 & 254 & 7.13 & 100 & 1170 \\
\hline Philodendron & 32.75 & 489 & 39.40 & 392 & 45.83 & 643 & 1524 \\
\hline Monstera & 1.00 & 15 & 3.02 & 30 & 3.56 & 50 & 95 \\
\hline Rhodospatha & 0.67 & 10 & 11.26 & 112 & 30.01 & 421 & 543 \\
\hline Stenospermation & 9.11 & 136 & - & 0 & - & 0 & 136 \\
\hline Syngonium & - & 0 & 7.24 & 72 & 12.47 & 175 & 247 \\
\hline Xanthosoma & 1.61 & 24 & 7.84 & 78 & 0.29 & 4 & 106 \\
\hline Chlorospatha & 0.20 & 3 & 2.01 & 20 & - & 0 & 23 \\
\hline Dieffenbachia & - & 0 & 3.72 & 37 & 0.71 & 10 & 47 \\
\hline Total & 100 & 1493 & 100 & 995 & 100 & 1403 & 3891 \\
\hline
\end{tabular}

El porcentaje de los individuos varía de acuerdo a los sectores en los que se realizó la investigación.

especies más abundantes de hábito hemiepífito fueron: Philodendron tenue K. Koch \& Augustin, Rhodospatha dodsonii Croat y Philodendron sulcatum K. Krause.

La Perla. Se encontraron 29 especies en 7 géneros (Anthurium, Philodendron, Rodospatha, Monstera, Xanthosoma, Dieffenbachia y Syngonium). El género con mayor representatividad fue Philodendron (643), la especie más abundante de hábito hemiepífito fue Philodendron sulcatum K. Krause. (Cuadro 1).

Abundancia de géneros. Se registraron 9 géneros correspondientes a: 30 Anthurium, 21 Philodendron, 4 Monstera, 2 Chlorospatha, Rhodospatha, Stenospermation y Xanthosoma, finalmente 1 Dieffenbaquia; para un total de 65 especies. (Figura 3)

Abundancia de Araceae por sector. El sector con mayor abundancia fue Sarapullo, debido al número elevado de individuos de los géneros Anthurium y Philodendron, le sigue el sector La Perla debido a la presencia de un alto número de individuos en el género Philodendron y Rhodospatha, por último, Peripa en el que también predomina un alto número de individuos del género Philodendron. (Figura 4)

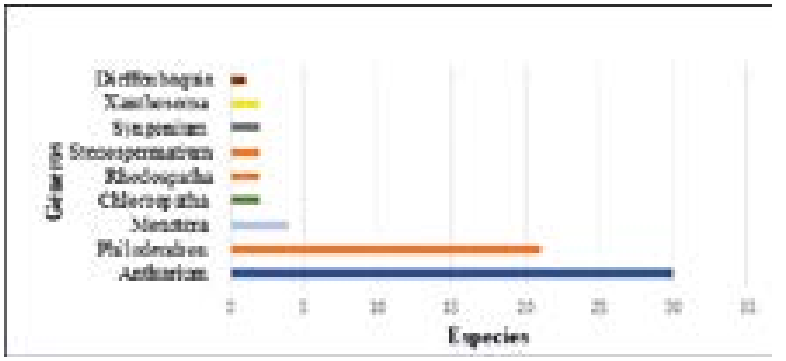

Figura 3. Abundancia de Géneros y especies de Araceae en los sectores Sarapullo, Peripa y La Perla

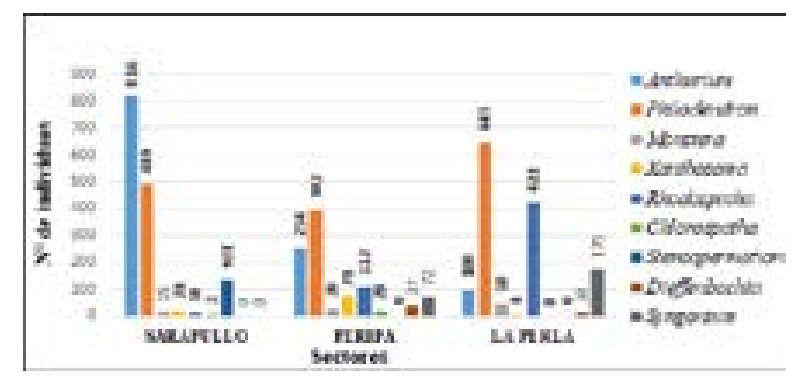

Figura 4. Abundancia de Araceae en base al número de individuos y a los sectores Sarapullo, Peripa y La Perla 


\section{Frecuencia de especies por sector}

Cuando una especie se encontró en los diez transectos de un sector se consideró como la más frecuentes, cuando se encontró en un solo transecto se consideró la menos frecuente.

En Sarapullo, 6 especies estuvieron presentes en los 10 transectos: Anthurium truncicolum Engler, Anthurium dolichostachyum Sodiro, Anthurium ocreathum Sodiro, Philodendron sulcatum K. Krause, Philodendron oligospermumn Engler y Philodendron fibrosum Sodiro ex. Croat. Especies que se presentaron una sola vez son: Philodendron pogonocaule Madison, Anthurium striatipes Sodiro, Philodendron beniteziae Croat, Anthurium mindense var. Mindense Sodiro.

En Peripa, 3 especies estuvieron presentes en los 10 transectos: Philodendron sulcatum K. Krause, Philodendron senatocarpium Madison $y$ Syngonium crassifolium (Engler) Croat. Especies que se presentaron una sola vez: Anthurium laciniosum Sodiro, Philodendron jadavisianum G. S. Bunting y Anthurium nigropunctatum Croat \& Rodríguez. En La Perla, 1 especie estuvo presente en los diez transectos: Philodendron clarkei Croat. Especie presentada una sola vez: Anthurium incomptum Madison (Cuadro 2).

Los géneros con hábito terrestre Xanthosoma y
Chlorospatha son más abundantes en Peripa, luego en Sarapullo y finalmente en La Perla; mientras que Philodendron y Rhodospatha de hábito hemiepífito se manifiestan más abundantes en La Perla y los géneros Anthurium y Stenospermation de hábito epífito se manifiestan más abundantes en Sarapullo, concluyendo que los géneros de hábitos hemiepífitos y epífitos se presentan en mayor porcentaje frente a los géneros de hábitos terrestres.

Los patrones de mayor distribución están relacionados con una alta precipitación, una baja evapotranspiración y una elevación intermedia entre 700 y 1500 msnm. Rodríguez 1987, Benavides, 1992a). Siendo obvia la disminución de especies y número de individuos en hábitats secos. En cuanto a la relación entre Araceae y su árbol hospedero, textura y forma, Benavides (1992b) comprobó que no existe una relación significativa, es decir su distribución es independiente de las características de los árboles.

Densidad. Sarapullo presenta 1.49 individuos por $\mathrm{m}^{2}$; La Perla 1.40 individuos por $\mathrm{m}^{2}$ y Peripa 0.99 individuos por $\mathrm{m}^{2 ;}$ la mayor densidad en Sarapullo se explica debido a la existencia de un grupo de especies dominantes con un gran número de individuos: Philodendron sulcatum

Cuadro 2. Frecuencia de especies en los 10 transectos en los sectores Sarapullo, Peripa y La Perla

\begin{tabular}{|c|c|c|c|}
\hline Sector & $\begin{array}{r}\text { Total, } \\
\text { Especies }\end{array}$ & $\mathbf{N}^{\circ}$ & Especies frecuentes en los 10 transectos \\
\hline \multirow{6}{*}{ Sarapullo } & \multirow{6}{*}{34} & 1 & Anthurium truncicolum Engler \\
\hline & & 2 & Anthurium dolichostachyum Sodiro \\
\hline & & 3 & Anthurium. ocreathum Sodiro \\
\hline & & 4 & Philodendron sulcatum K. Krause \\
\hline & & 5 & Philodendron oligospermun Engler \\
\hline & & 6 & Philodendron. fibrosum Sodiro ex. Croat. \\
\hline \multirow{3}{*}{ Peripa } & \multirow{3}{*}{36} & 1 & Philodendron sulcatum K. Krause \\
\hline & & 2 & Philodendron senatocarpium Madison \\
\hline & & 3 & Syngonium crassifolium (Engler) Croat. \\
\hline La Perla & 29 & 1 & Philodendron clarkei Croat \\
\hline
\end{tabular}


Cuadro 3. Valores de diversidad Simpson y Shannon Wiener en los sectores Sarapullo, Peripa y La Perla

\begin{tabular}{lllllll}
\hline Sitio & Géneros & Especies & Individuos & $\begin{array}{l}\text { Diversidad } \\
\text { Simpson } \\
\text { (1-D) }\end{array}$ & $\begin{array}{l}\text { Diversidad } \\
\text { Shannon } \\
\text { Wiener } \\
\text { (base Log } \\
\text { nat.) }\end{array}$ & $\begin{array}{l}\text { Interpretación de } \\
\text { los índices } \\
\text { (basada en } \\
\text { Yánez, 1997) }\end{array}$ \\
\hline Peripa & 8 & 36 & 995 & 0.93 & 2.99 & Alta \\
Sarapullo & 7 & 34 & 1493 & 0.92 & 2.81 & Mediana \\
La Perla & 7 & 29 & 1403 & 0.82 & 2.30 & Mediana \\
& & & & & & \\
\hline
\end{tabular}

K. Krause, Anthurium Cordiforme Sodiro, Anthurium dolichostachyum Sodiro, Anthurium interruptum Sodiro, y Stenospermation densiovulatum Engler; que cubren el $44.47 \%$ del total. Para Peripa (con la menor densidad), las especies dominantes son: Philodendron sulcatum K. Krause, Philodendron tenue K. Koch \& Augustín, Anthurium versicolor Sodiro y Rhodospatha dodsonii Croat; cubren el $42.8 \%$ del total.

Las dominancias de los géneros Anthurium, Philodendron y Rhodospatha en el caso La Perla de hábitos epífitos y hemiepífitos, están determinando los valores de densidad obtenidos. También podrían explicarse los resultados de densidad por los niveles de conservación de los sectores; en Peripa existe un extractivismo selectivo pues allí el grupo étnico Tsáchila; realiza uso de Araceae y especies asociadas a esta familia.

Índices de Diversidad. Los índices de diversidad (Simpson en su forma 1-D y Shannon-Wiener en base a logaritmo natural), produjeron la siguiente información. (Cuadro 3).

Los índices se ven incrementados en Peripa y Sarapullo, quizá debido a las estructuras de los bosques que presentan doseles más abiertos con mayor número de árboles, permitiendo mayor ingreso de luz para epifitación, germinación y crecimiento de Araceae, en el caso de La Perla el dosel es más cerrado y el número de árboles es menor por lo que se vería dificultada la instalación de especies epífitas y hemiepífitas.

Además la aproximación a las elevaciones intermedias óptimas para el desarrollo de esta familia, al igual que el clima de moderada temperatura y baja evapotranspiración para el desarrollo del género Anthurium, marcan un incremento de la diversidad, como lo demuestran los sectores de Peripa y Sarapullo; mientras que su alejamiento a estas variables, caso $\mathrm{La}$ Perla, determinan la disminución, lo que permite sugerir que una de las variables determinantes para la diversidad de esta familia es la altitud.
Curvas de Abundancia - Diversidad. Las curvas fueron construidas utilizando datos totales de cada sitio) (la construcción de los vectores está basada en Magurran, 1989 citado en Yánez, 1997) (Figura 4).

Según este modelo interpretativo de diversidad, en el eje $\mathrm{X}$ se representa la riqueza de especies y en el eje $\mathrm{Y}$, la proporción de individuos con los que cada especie aporta al sitio

Un sector con la más alta diversidad se encontraría representado por un vector lo más largo horizontalmente (eje X) y lo más alto posible (eje Y). Esto es lo que sucede con el vector correspondiente a Peripa, que demuestra alta diversidad, confirmando los datos obtenidos por los índices de diversidad.

Cuando la riqueza de especies y la abundancia de individuos por especie produce vectores que se ajustan a este modelo, los vectores corresponden a sistemas o comunidades en los cuales existen muy pocas especies dominantes (una, dos o tres), un número moderado de especies codominantes y relativamente pocas especies raras (Yánez, 2004).

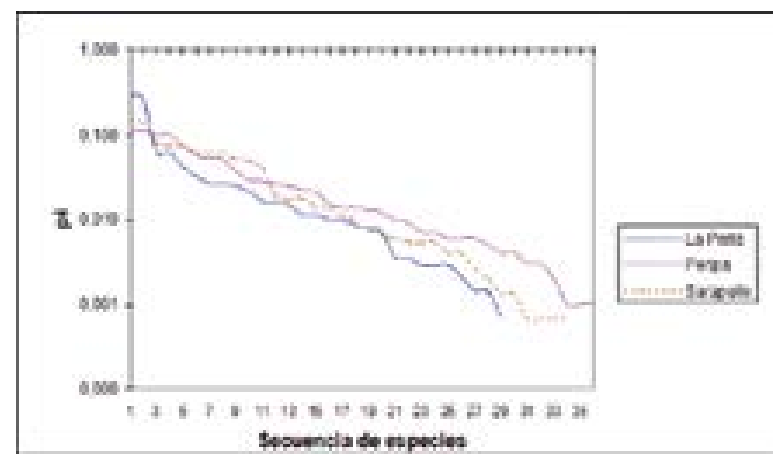

Figura 5. Curvas de Abundancia-Diversidad para los tres sectores estudiados (a) La Perla, (b) Peripa, (c) Sarapullo 
Especies dominantes por sector y en general. En efecto, como se pudo observar; En sarapullo, solo 2 especies (Philodendron sulcatum K. Krause $y$ Anthurium cordiforme Sodiro) presentan muchos individuos (cada una con más del $10 \%$ ), mientras que la mayoría de las especies (20) contribuyen con menos del 2\% de individuos, entre ellas Anthurium mindense var. Mindense Sodiro, Anthurium striatipes Sodiro, Philodendron beniteziae Croat y Philodendron pogonocaule Madison.

En Peripa, solo 4 especies (Philodendron tenue K Koch \& Augustin, Philodendron sulcatum K Krause, Rhodospatha dodsonii Croat y Anthurium versicolor Sodiro) presentan muchos individuos (cada una coopera con el $10 \%$ o más del $10 \%$ ), la mayoría de especies (20) cooperan con menos del 2\%, entre ellas están Anthurium laciniosum Sodiro, Philodendron jodavisianum G. S. Bunting y Monstera dubia (Kunth) Engler.

En La Perla, las especies más abundantes son Philodendron sulcatum $\mathrm{K}$ Krause y Rhodospatha dodsonii Croat (con más del $25 \%$ de aporte a la comunidad por cada una), mientras que las especies que contribuyen con menos del $2 \%$ son unas 16 , entre ellas: Anthurium incomptum Madison, Anthurium nigropunctatum Croat \& Rodríguez y Anthurium obtusum (Engler) Grayum. (Figura 6).

Como se aprecia en la Figura 5, Philodendron sulcatum K Kraus, es la especie dominante en los tres sectores y podría considerarse como generalista, aunque también su dominancia se puede deber a un tipo de protección o aislamiento por la ubicación en microclimas en los bosques que ocupa, como es el caso del Sector La Perla, que se encuentra bajo un régimen de conservación y por supuesto guarda esos microclimas delimitados por algún tipo de barrera climática, que ayudan a su conservación.

Similitud entre sectores. El coeficiente de similitud de Sorensen se basó en los datos de presencia-ausencia de especies en cada sector, poniendo énfasis en las especies compartidas, mientras que el índice de similitud de Morisita considera, además la cantidad de individuos que aportan a dicha presencia ausencia. De acuerdo con

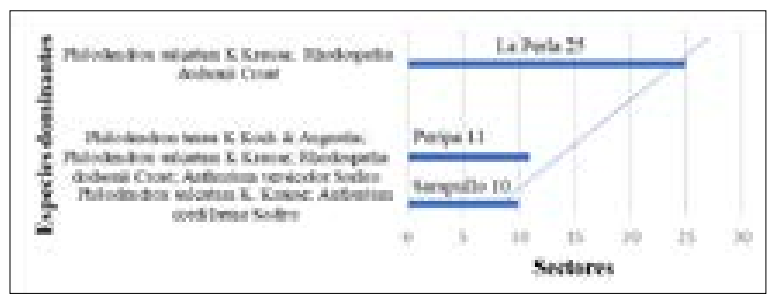

Figura 6. Especies dominantes en los sectores La Perla, Peripa, Sarapullo ello mayor grado de similitud se encontró entre PeripaLa Perla, con 25 especies en común, luego está el par Peripa-Sarapullo con 9 especies en común y en tercer lugar Sarapullo-La Perla con 6 especies en común. Finalmente, la similitud entre los tres sectores cuenta con 5 especies en común (Cuadro 4).

Como se aprecia en el Cuadro 4, Philodendron sulcatum K. Krause, junto con otras especies, son las que se encuentran en los tres sectores con mayor frecuencia, esto quizá se debe como se dijo con anterioridad a que son especies generalistas. La ubicación de Peripa con un nivel altitudinal intermedio con relación a los otros sectores del estudio, le permite ser el sector que mayor número de especies comparte con los otros dos, por ejemplo, con La Perla ya que la cercanía geográfica y el compartir variables climáticas, probablemente determinan el alto número de compartición; mientras que Sarapullo que está separado con los otros dos tanto a nivel climático como altitudinal, no comparte muchas especies. Esta diferencia altitudinal sumada a variaciones de la temperatura media y a variaciones de precipitación, permiten ubicar a Sarapullo como parte de una zona de vida diferente.

\section{Similitud entre transectos}

Se efectuó un Dendrograma de frecuencias basado en los 30 transectos analizados, lo que arrojó una buena representación de la diversidad entre sitios mediante el análisis Clúster (Análisis de Clasificación) (Figura 7).

\section{Cuadro 4. Especies compartidas entre Sarapullo, Peripa, La Perla}

\begin{tabular}{|c|c|c|c|c|}
\hline $\mathbf{N}^{\circ}$ & Especie & Sarapullo & Peripa & $\begin{array}{c}\text { La } \\
\text { Perla }\end{array}$ \\
\hline 1 & $\begin{array}{l}\text { Philodendron } \\
\text { sulcatum } \mathrm{K} . \\
\text { Krause }\end{array}$ & 419 & 101 & 419 \\
\hline 2 & $\begin{array}{c}\text { Anthurium } \\
\text { dolichostachyum } \\
\text { Sodiro }\end{array}$ & 38 & 14 & 38 \\
\hline 3 & $\begin{array}{l}\text { Anthurium } \\
\text { versicolor } \\
\text { Sodiro }\end{array}$ & 16 & 100 & 16 \\
\hline 4 & $\begin{array}{c}\text { Xanthosoma } \\
\text { daguense Engler }\end{array}$ & 4 & 75 & 4 \\
\hline 5 & $\begin{array}{c}\text { Monstera } \\
\text { adansonii Schott }\end{array}$ & 12 & 4 & 12 \\
\hline
\end{tabular}



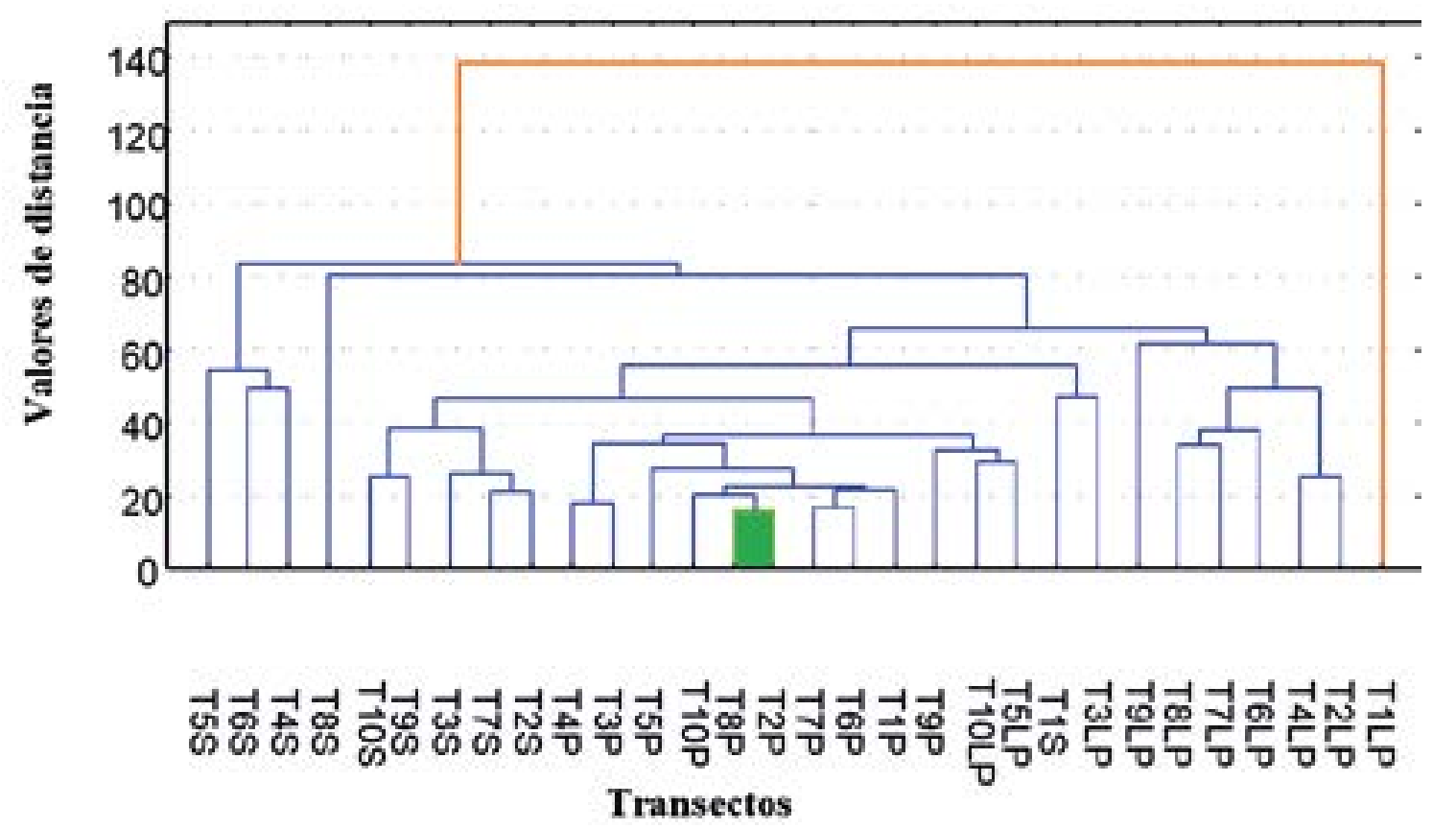
Figura 6. Dendrograma para los 30 transectos de muestreo en base a su composición florística de
Araceae (Método de la Unión Promedio en base a distancias euclidianas)

Mientras más baja es la unidad de distancia a la que se unen dos entidades más similares son en su composición de Araceae, por ejemplo, las unidades más similares de entre todas son T8P y T2P, a excepción del transecto T1LP en el Sector La Perla, que corresponde a un sitio muy alterado, cerca del que se iniciaron los transectos.

Del total de 65 unidades de distancia, existen 5 clados, que se unen más rápido entre sí, en los transectos pertenecientes a Peripa y La Perla y luego poco después se unen con los de Sarapullo. Esto refuerza lo discutido en párrafos anteriores sobre la mayor similitud de los dos sectores.

\section{Conclusiones}

$\mathrm{E}^{\mathrm{I}}$ total colectado en los tres sectores fue de 3891 individuos; con 1493 para Sarapullo, 995 para Peripa y 1403 para La Perla. La abundancia genérica está determinada por Anthurium en el sector de Sarapullo; mientras Philodendron alcanza su mayor representación en el sector La Perla y en Peripa pero seguido muy de cerca por el género Anthurium; lo que permite además concluir que el género Anthurium muestra preferencias de hábitats con alturas intermedias, con climas de moderada temperatura y bajas evapotranspiraciones; mientras que el género Philodendron alcanza su mayor representación en los sectores de Peripa y La Perla; como puede verse entonces, las preferencias de hábitats de los principales géneros de la familia se expresan en condiciones opuestas.

Los hábitos en la familia Araceae son una condición que influye en su diversidad o en su abundancia. Los hábitos hemiepífitos y epífitos se presentan en mayor porcentaje frente a los hábitos terrestres. Por ejemplo, Philodendron sulcatum K. Krause de hábito hemiepífito se encuentra en los tres sectores abundantemente. Podríamos añadir que las especies con hábitos hemiepífitos ecológicamente se encuentran más aptas para desarrollarse o competir con otras especies generalistas.

La distribución de las especies de la familia Araceae está íntimamente relacionada a los factores ecológicos de los diferentes sectores, de ahí que la similitud de los mismos en base a la composición florística es más fuerte entre los sectores de Peripa y La Perla localizadas en rangos de altura no muy distantes lo que les hace además compartir las variables climáticas. El sector de Sarapullo presenta una composición florística más semejante a Peripa que a La Perla probablemente por tener diferencias altitudinales inferiores con relación a La Perla.

Algunas especies de Araceae tienden a ser especialistas de hábitat, restringidas a una sola zona de vida; contrariamente, otras especies no prefieren hábitat en particular y pueden estar hasta en tres zonas de vida. 
En general, la mayoría son de distribución relativamente amplia y toleran disturbios considerables, siempre y cuando las áreas no hayan sido quemadas.

La saturación de especies para la Familia Araceae en los sectores de estudio se alcanza a los $800 \mathrm{~m}^{2}$; sin embargo, los sectores de Peripa y La Perla se manifiestan como ambientes muy heterogéneos al presentar especies fuera de los 10 transectos, cabe anotar que el límite de colecciones para Araceae de hábitos epífitos fue de $5 \mathrm{~m}$ de altura, lo que podría llevar a disminuir el número de algunas especies que pudieron epifitar a mayor altura y sesgar de alguna manera la información.

Es más probable que las distribuciones restringidas observadas entre las especies endémicas de la región andina se deban a especializaciones a los microclimas en los bosques que ocupan, que a la falta de potencial para dispersión entre diferentes áreas. Sin embargo, es cierto que hay especies, géneros o familias que muestran un endemismo, caracterizado por poblaciones delimitadas por barreras geográficas.

Las características ambientales de Sarapullo determinadas por su altitud sobre el nivel del mar permiten un mayor desarrollo de especies del género Anthurium; considerando además que conforme aumenta la altura, las epífitas aumentan en número, mientras que en las zonas más bajas es el género Philodendron el que tienen mayores oportunidades.

Las Araceae de hábitos hemiepífitos no tienen una especificidad con el sustrato o con las características del substrato lo que hace presumir que la dominancia del hábito hemiepífito en la familia estaría más bien relacionado con la necesidad quizás de disponibilidad de luz, la estratificación del bosque, o el diámetro del tronco (disponibilidad de espacio) entre otros.

Los 4 nuevos registros de Araceae encontrados (Philodendron sp. nov. 1 y 2 en Sarapullo, Philodendron sp. nov 3 en Peripa y Rhodospatha sp nov. 4 en Sarapullo) demuestran que la realización de nuevos inventarios en lugares menos explorados es importante para conocer más acerca de su diversidad, su ecología y su aporte a la flora en general.

\section{Bibliografía}

Aad Van Ufellen, 1996, Creative Flower Arringing with Anthurium, Kosmos Z\&K publishers, Utrecht/ Antwerpen, Holland.

Alarcón., R. 1984, Etnobotánica de los Quichuas del Oriente Ecuatoriano, Ediciones del Banco Central del Ecuador, Quito.

Avances en Ciencias e Ingenierías, 2015, Vol. 7, No. 1, Págs. B39-B87. Catálogo de especímenes tipo del Herbario Nacional del Ecuador (QCNE), Museo Ecuatoriano de Ciencias Naturales. Diana Fernández-Fernández, Efraín
Freire M., Marcia Peñafiel C., Giovanna Romero, Fanny Tello y Elsa Toapanta.

Benavides, G. 1992a. Las Araceae de la Estación Forestal LA FAVORITA, Tesis de Licenciatura. Escuela de Biología de la Universidad Central del Ecuador.

Benavides, G. 1992b. Especificidad en las hemiepífitas de la Familia Araceae con los árboles de bosque primario de la Estación Biológica Jatun Sacha. Memorias del Curso de Post Grado Ecología de Poblaciones y Biología de la Conservación.

Cerón, C. 1973., Etnobotánica Quichua en la vía Hollín Loreto, Prov. de Napo Hombre y Ambiente 25., ABYAYALA. Quito, pags. 131-171

Cerón, C. 1993. La Vegetación en La Reserva Forestal La Favorita. Rev. Cátedra No. 3. Editorial Universitaria. Quito-Ecuador.

Croat, T. 1985. Collecting and preparing Specimens of Araceae. Armais. Missouri Bot. Garden. 72: 252-258.

Dodson, C.H. \& A.H. Gentry. 1978. Flora of the Río Palenque Science Center, Los Ríos Province, Ecuador. Selbyana. 4 (1-6): 1-628.

Dodson, C.H. \& A.H. Gentry \& F.M. Valverde. 1985. Flora de Jauneche, Los Ríos Province, Ecuador. Ediciones Banco Central del Ecuador (1-6): 1-512.

EcoCiencia, 1986. Plan de Manejo del Bosque Protector la Perla

Ganzenmüller, A., F. Cuesta-Camacho, M. G. Riofrío, C. González y F. Baquero. 2010. Caracterización ecosistémica y evaluación de efectividad de manejo de los bosques protectores y bloques del Patrimonio Forestal ubicados en el sector ecuatoriano del Corredor de Conservación Chocó-Manabí. MAE, EcoCiencia y CI. Quito.

Gómez, D \& G, Toaza. 1997. Composición Florística de Remanentes de Bosques en el Noroccidente de la provincia de Pichincha. FAO. Quito-Ecuador.

Gobierno Autónomo Descentralizado Parroquial de La Concordia. 2016. Plan Piloto de Desarrollo y Ordenamiento Territorial

León-Yánez, S., R. Valencia, N. Pitman, L. Endara, C. Ulloa Ulloa \& H. Navarrete (eds.). 2011. Libro rojo de las plantas endémicas del Ecuador, $2^{\mathrm{a}}$ edición. Publicaciones del Herbario QCA, Pontificia Universidad Católica del Ecuador, Quito

Ministerio del Ambiente del ecuador., 2015. Subsecretaría de patrimonio natural. Sistema único de información ambiental unidad de procesamiento de información y Geomática. Sistema nacional de monitoreo de patrimonio natural. Estadísticas de patrimonio natural. Datos de bosques, ecosistemas, especies, carbono y deforestación del Ecuador continental.

Ministerio de Ambiente del Ecuador (2013). Sistema de Clasificación de los Ecosistemas del Ecuador Continental. Quito, Ecuador, Subsecretaría de Patrimonio Natural. 
Ministerio del Ambiente del Ecuador. 2105, mapa histórico de deforestación. Unidad de Monitoreo. Página 108

Madison, M. 1978b. The Species of Anthurium with Palmately divided leaves. Selbyana, 2(2-3): 239-282.

Rodríguez, J. 1987, Estudio de la Familia Araceae y Taxonomía del género Anthurium en la Reserva Endesa, Occidente de la Prov. De Pichincha, PUCE, Depto. de Ciencias Biológicas. Tesis de Licenciatura, QuitoEcuador. 168pp.

Sierra, R. 2013. Patrones y factores de deforestación en el Ecuador continental, 1990-2010. Y un acercamiento a los próximos 10 años. Conservación Internacional Ecuador y Forest Trends. Quito, Ecuador

Yánez, P., 1997. Análisis de la distribución de especies vegetales a lo largo de un gradiente altitudinal páramo, selva nublada del Parque Nacional Sierra Nevada, Venezuela Universidad de los Andes. Tesis para obtener el título de Magíster Cientiae en ecología Tropical.

Yánez, P. 2004. Memorias del Seminario-Taller sobre Biometría y Bioestadística Fundamentales. Agosto 2004, Colegio Nacional Galápagos. Puerto Ayora--Ecuador 